\title{
$8 \mathrm{H}-, 10 \mathrm{H}-$, $14 \mathrm{H}-\mathrm{SiC}$ formation in $6 \mathrm{H}-3 \mathrm{C}$ silicon carbide phase transitions
}

\author{
S.I. Vlaskina ${ }^{1,2}$, G.N. Mishinova ${ }^{3}$, V.I. Vlaskin ${ }^{4}$, V.E. Rodionov ${ }^{1}$, G.S. Svechnikov ${ }^{1}$ \\ ${ }^{1}$ Institute of Semiconductor Physics, National Academy of Science of Ukraine \\ 45, prospect Nauky,03028 Kyiv, Ukraine; e-mail: businkaa@mail.ru \\ ${ }^{2}$ Yeoju Institute of Technology, 200 Myeongseong-ro, Gyeonggi-do, 469-705 Korea \\ ${ }^{3}$ Taras Shevchenko Kyiv National University, 64, Volodymyrska str., Kyiv 03033, Ukraine \\ ${ }^{4}$ Sensartech, 2540 Lobelia Dr., Oxnard, 93036 California, USA
}

\begin{abstract}
In this paper the results of photoluminescence researches devoted to phase transitions in $6 \mathrm{H}-3 \mathrm{C}-\mathrm{SiC}$ have been presented. High pure $6 \mathrm{H}-\mathrm{SiC}$ crystals grown by Tairov's method with and without polytype joint before and after plastic deformation at high temperature annealing were investigated using optical spectroscopy. Low temperature photoluminescence changes in the transition phase of $\mathrm{SiC}$ crystal represented with the stalking fault spectra within the temperature range 4.2 to $35 \mathrm{~K}$. The stalking fault spectra indicate formation of metastable nanostructures in $\mathrm{SiC}$ crystals $\left(14 \mathrm{H}_{1}\right.$ $\left.\langle 4334\rangle, 10 \mathrm{H}_{2}\langle 55\rangle, 14 \mathrm{H}_{2}\langle 77\rangle\right)$. The phononless part of each stalking fault spectrum consists of two components of radiative recombination that are responsible for hexagonal and cubic arrangement of atoms. Each of radiative recombination components in the stalking fault spectrum has the width of entire band $34 \mathrm{meV}$ and shifts relative to each other by $26 \mathrm{meV}$. The overlap area of those components equals to $8 \mathrm{meV}$. The super-fine structure of the recombination components in spectrum is observed, and it is related to different $\mathrm{Si}-\mathrm{Si}$ or $\mathrm{C}-\mathrm{C}$ and $\mathrm{Si}-\mathrm{C}$ bonds. Behavior of all the stalking fault spectra is similar (temperature, decay of luminescence). The processes of the phase transition are explained by the mechanism of interfacial rearrangements in the $\mathrm{SiC}$ crystals.
\end{abstract}

Keywords: silicon carbide, polytype, stacking fault, nanoparticle, phase transitions.

Manuscript received 10.06.13; revised version received 26.07.13; accepted for publication 19.09.13; published online 30.09.13.

\section{Introduction}

Researches associated with formation of polytype structures are a staple of modern condensed matter physics of nanostructures. The phenomenon of polytypism is inherent to single crystals, films, powders, polycrystalline compact materials, and nanostructures. Polytypes of $\mathrm{SiC}$ differ fundamentally only by the number and the sequence of position of the atomic $\mathrm{Si}-\mathrm{C}$ bilayers relative to the adjacent (neighboring) bilayers in a cubic (c) or hexagonal (h) arrangements. Replacement of any layer in the cubic setting on a hexagonal (or vice versa) can be viewed as a stacking fault (SF) of the polytype, which has a strictly defined motif of alternating atomic planes, the so-called zig-zag chains. Regular input of hlayers in a cubic structure $3 \mathrm{C}$-SiC ( $\beta$-phase) can give structure of the $\alpha$-phase (H or R).

In terms of the dislocation, the transition mechanism of the cubic structure to the hexagonal structure is discussed in the articles [1-7]. In terms of formation of the multilayers polytypes, transition of the hexagonal structure to the cubic one is discussed in [813]. The interface interactions between $3 \mathrm{C}-\mathrm{SiC}$ and $2 \mathrm{H}-$ $\mathrm{SiC}$ have been also investigated [14]. The mechanism of this transition is the object of various simulations $[15,16]$. 
Also, SiC polytypes can be described by different stacking of $\mathrm{Si}-\mathrm{C}$ layers that are perpendicular to the direction of the SiC closed-packed plane. SF play the main role in the process of structure transformation $[11,13]$. SF dimension of a few $\mathrm{SiC}$ lattices is a quantum well $3 \mathrm{C}-\mathrm{SiC}$ within wider band-gap $\alpha-\mathrm{SiC}$. The structure leads to appearance of the quantum effects and $\mathrm{SiC}$ actually becomes a direct band gap semiconductor, which results in intense photoluminescence in the blue part of the spectrum. A new type of heterostructure can be created using $\mathrm{SiC}$, namely: heterostructure not between different materials but between different modifications of the same material [17]. Degradation of the electrical characteristics of bipolar $\mathrm{SiC}$ devices is explained by the presence of SF in crystal bulk [18, 19]. Formation of different polytypes under the same thermodynamic conditions can be understood when using optical spectroscopy analysis $[1,20]$.

This work is aimed at properties of high purity $6 \mathrm{H}-$ SiC crystals (before and after plastic deformation and high temperature annealing treatment) and disordered grown layers in the crystals of $\alpha-\mathrm{SiC}$ (mainly $6 \mathrm{H}-\mathrm{SiC}$ ) by using optical spectroscopy such as low temperature photoluminescence (LTPL), Photoluminescence excitation spectra (PES) and absorption spectra have been analyzed.

\section{Experiment}

The luminescence spectra were obtained using excitation by the mercury lamp $(\lambda=365 \mathrm{~nm})$, and by lasers: nitrogen laser $(\lambda=337 \mathrm{~nm})$; He-Cd laser $(\lambda=441.6 \mathrm{~nm})$; $\operatorname{Ar}-$ laser $(\lambda=488 \mathrm{~nm})$.

Crystals were grown by sublimation (Lely method), were selected according to the phase composition $(\alpha-$ or $\beta$-phase) and the degree of structural disorder (control, twinning, and one-dimensional disorder). Crystal structural researches were carried out using X-ray diffraction (Laue method) and electron diffraction methods. Thermal treatment of crystals (hightemperature annealing) was performed in a resistance furnace with a graphite heater in argon atmosphere at $T=2000 \ldots 2100{ }^{\circ} \mathrm{C}$ for 1 to 10 hours. Plastic deformation of the samples was carried out in a resistance furnace at the argon atmosphere by three points bending at $2000{ }^{\circ} \mathrm{C}$ for $15 \ldots 30 \mathrm{~min}$.

\section{1. $6 H$-SiC perfect crystals}

High purity crystals with a very low concentration of impurities have been selected for the spectroscopic investigation of the phase transition $6 \mathrm{H}-3 \mathrm{C} \mathrm{SiC}$. Laue diffraction patterns indicated a single-phase crystal composition. Normally, only in such perfect crystals of $6 \mathrm{H}-\mathrm{SiC}$ observed is the well known spectrum of exciton impurity (nitrogen) complexes (as known as PRS) together with ABC-spectra associated with titanium. The spectra of LTPL of the crystal (at $4.2 \mathrm{~K}$ ) with the non-compensated nitrogen concentration
$N_{D}-N_{A} \sim(6 \ldots 7) \cdot 10^{16} \mathrm{~cm}^{-3}$ were investigated. The change of the LTPL spectra had been observed after plastic deformation at the temperature from 2000 up to $2100{ }^{\circ} \mathrm{C}$ for $30 \mathrm{~min}$ in argon atmosphere. A fine spectrum structure of the $\mathrm{SiC}$ appeared. Similar results in $\mathrm{SiC}$ crystal after annealing were demonstrated and specified as SF in Refs. [1, 9, 13].

The intensity of the PRS and ABC maxima is considerably reduced after applying the force to the crystals. The plastic deformation stimulates an emergence of the so-called L-spectrum, which is associated with presence of vacancies, and it was previously obtained only by the ion bombardment of crystals. This confirms a possibility of vacancies formation during the process.

\subsection{Grown polytype's transformations in SiC crystals}

For better understanding the process of transformation the pure $\mathrm{SiC}$ crystals $\left(N_{D}-N_{A} \sim(4 \ldots 9) \cdot 10^{16} \mathrm{~cm}^{-3}\right)$ with natural inter-grown $\mathrm{SiC}$ polytype's joints were specially selected. The crystals have been studied in detail for the presence of similar spectra. Crystal structure of disordered layers was determined by diffuse smearing of reflections in Laue pattern. The "twin plates" of the $\beta$ phase in $\mathrm{SiC}(10 \ldots 100 \AA$ lamellae $)$ and the basic structures of the $\alpha$-phase: $6 \mathrm{H}-\mathrm{SiC}, 15 \mathrm{R}-\mathrm{SiC}, 21 \mathrm{R}-\mathrm{SiC}$ were investigated. Fig. 1 shows LTPL of various samples of the inter-grown polytypes at $T=4.2 \mathrm{~K}$. The overall spectral pattern of each sample represents a specific series of spectra $\mathrm{SF}_{1}$ (Fig. 1a), $\mathrm{SF}_{2}$ (Fig. 1a, c), $\mathrm{SF}_{3}$ (Fig. 1a, b), $\mathrm{SF}_{4}$ (Fig. 1b) which look like spectra of the $6 \mathrm{H}-\mathrm{SiC}$ crystal after applying the force.
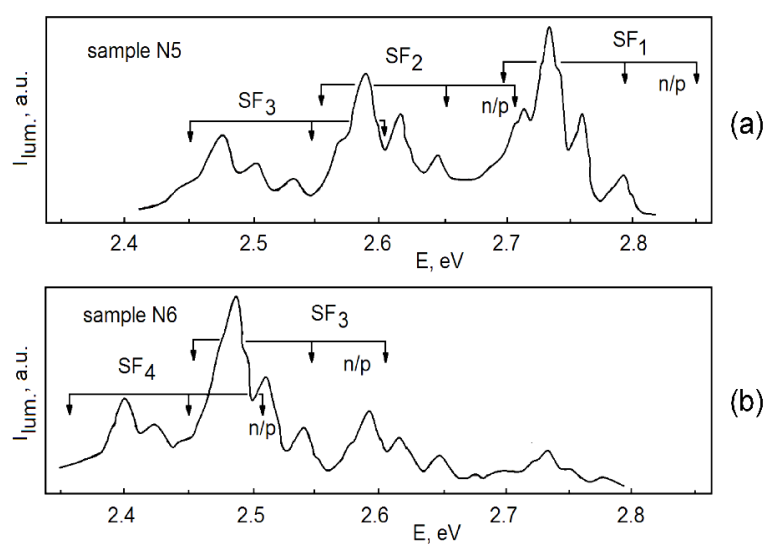

(b)

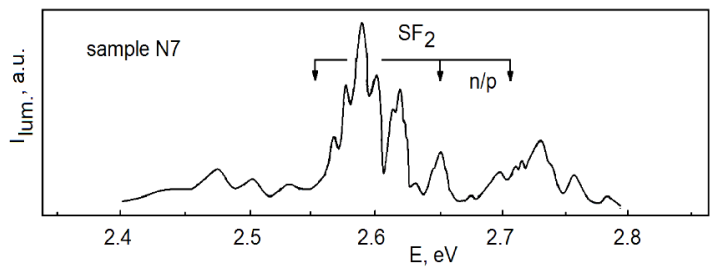

Fig. 1. LTPL at $4.2 \mathrm{~K}$ of the inter-grown polytypes joint in different samples: (a) sample N5; (b) sample N6; (c) sample N7. 


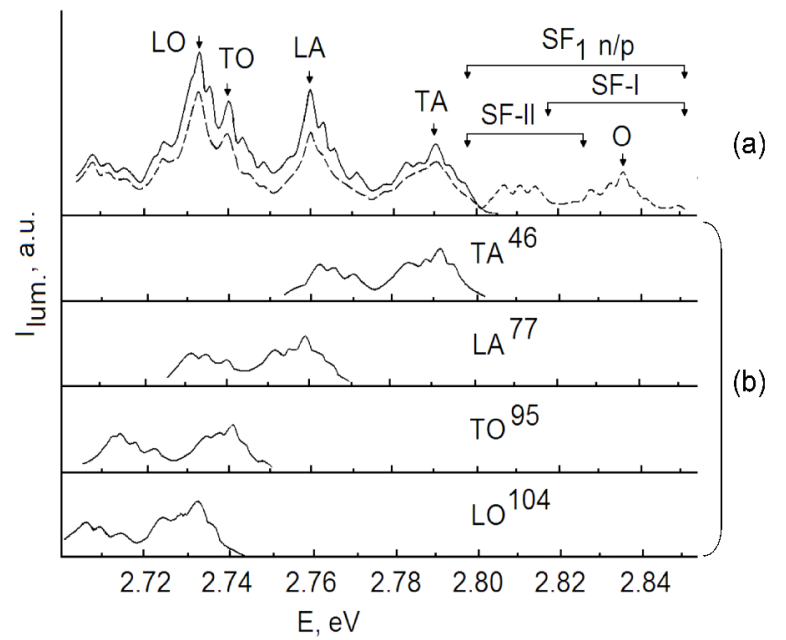

Fig. 2. $\mathrm{SF}_{1}$ spectrum of $\mathrm{SiC}$ at $4.2 \mathrm{~K}$ : (a) reconstituted phononless part of $\mathrm{SF}_{1}$ spectrum; (b) phonons in $\mathrm{SiC}$ involved in $\mathrm{SF}_{1}$ spectrum.

The difference is that all the SF fine spectra are located at the different energy scale with different intensities of the spectra. But a comprehensive spectroscopic analysis showed that all SF spectra have the same characteristics and principle of their construction regardless of placement in the common energy scale. Transcript structure of the spectrum $\mathrm{SF}_{i}$ (where $i=1,2,3,4$ ) is made with the spectrum $\mathrm{SF}_{1}$ that is most frequently observed. Transcripts of another $\mathrm{SF}_{i}$ fine spectrum are the same. Other $\mathrm{SF}_{i}$ spectra are located in different energy region compare with $\mathrm{SF}_{1}$ fine spectra.

The entire structure of the spectrum SF (Fig. 2a) can be obtained by summing together additive phonon replicas to some phononless part of the spectrum (Fig. 2b).

Phonons of extended edge Brillouin zone of the silicon carbide $\left(E_{\mathrm{TA}}=46 \mathrm{meV}, E_{\mathrm{LA}}=77 \mathrm{meV}, E_{\mathrm{TO}}=\right.$ $95 \mathrm{meV}, E_{\mathrm{LO}}=104 \mathrm{meV}$ ) are involved in the emission. Adding the phonon replicas spectrum to the proposed phononless structure spectrum gives the photoluminescence spectrum (LTPL) (Fig. 2a). By the way, a specific feature is that the phononless part itself is not observed. The phononless part of fine spectra was created in accordance with the structure of TA-replica and its transitions as a whole to higher energies by the energy of TA phonon $(46 \mathrm{meV})$. Fig. 3 shows that the phononless spectrum of $\mathrm{SF}_{1}$ is located within the energy region $(2.853 \ldots 2.793 \mathrm{eV})$ with the width of the entire band $60 \mathrm{meV}$. The energy region for SF - I spectrum is $2.853 \ldots 2.819 \mathrm{eV}$ and for $\mathrm{SF}-\mathrm{II}$ spectrum is $2.827 \ldots 2.793 \mathrm{eV}$, accordingly.

It turns out that, after determining the position of the phononless part of $\mathrm{SF}_{1}$ in the energy scale, the energy of the short-wave spectrum $\mathrm{SF}_{1}$ is the same as the exciton band gap of the SiC-polytype 21R-SiC at $T=$ $4.2 \mathrm{~K}$, and equal to $2.853 \mathrm{eV}$ [1]. The energy of inserting a $2 \mathrm{H}(3 \mathrm{C})$ crystal in a $3 \mathrm{C}(2 \mathrm{H})$ matrix had been calculated and is $68.4 \mathrm{meV} /$ atom for $(2 \mathrm{H})$, and $66.9 \mathrm{meV} /$ atom for (3C) [14]. Both calculated energy values are about the same as the energy obtained from a width of the entire band of $\mathrm{SF}_{i}$ spectra $(60 \mathrm{meV})$.

Careful investigation of phononless part of the $\mathrm{SF}_{1}$ showed that the spectrum by itself consists of two components are SF - I $(2.853 \ldots 2.819 \mathrm{eV})$ and $\mathrm{SF}-\mathrm{II}$ $(2.827 \ldots 2.793 \mathrm{eV})$. Each of them has the width of the entire band $34 \mathrm{meV}$. Maximums of SF - I and SF - II are shifted relatively to each other by $26 \mathrm{meV}$ (Fig. 4). The overlap spectrum area equals to $8 \mathrm{meV}$.

Two parts of the $\mathrm{SF}_{i}(\mathrm{SF}-\mathrm{I}$ and $\mathrm{SF}-\mathrm{II})$ spectrum also have super-fine structure. Resolution of the superfine structure SF - I and SF - II is different in the case of different natural inter-grown $\mathrm{SiC}$ crystal polytypes. The smallest width at half maximum of the fine structure $\mathrm{SF}-\mathrm{I}$ and SF - II components is $1.5 \mathrm{meV}$. According to Ref. [14], the process of interface formation in the $\mathrm{SiC}$ crystal requires energy $0.151 \mathrm{eV} / \AA^{2}$. This energy induces distortion of tetrahedrons and generates compressed $\mathrm{Si}-\mathrm{Si}$ and stretched $\mathrm{C}-\mathrm{C}$ bonds. So, the super-fine structures of SF - I and SF - II can be caused by the arrangement of each atom.

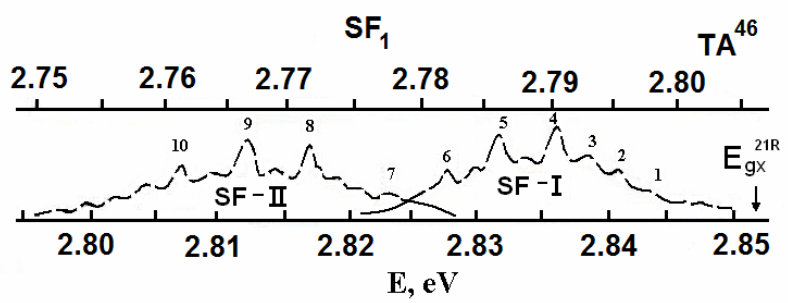

Fig. 3. The phononless part of the $\mathrm{SF}_{1}$ spectrum.

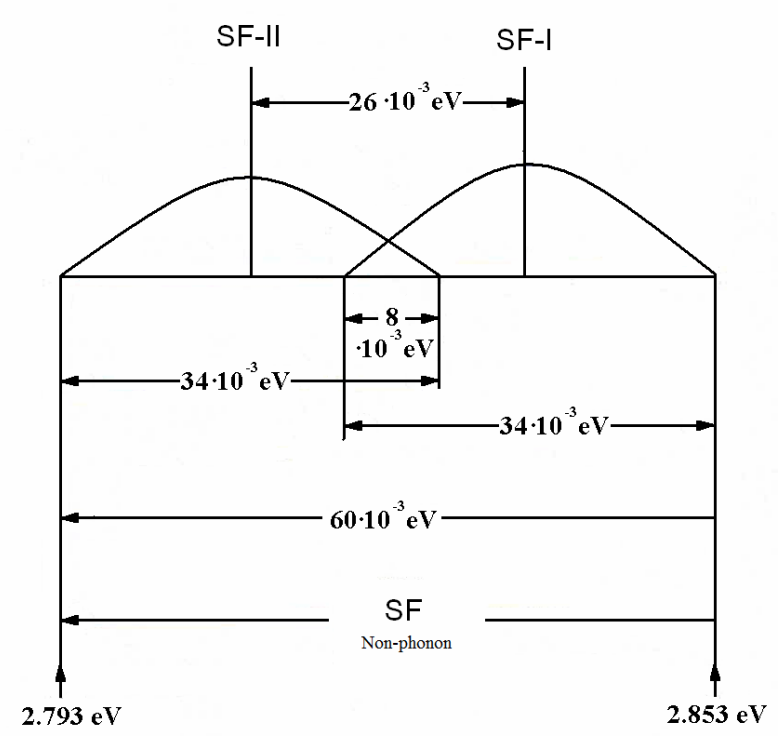

Fig. 4. Scheme of the phononless spectrum $\mathrm{SF}_{1}$. 


\section{Discussion}

Silicon carbide is a covalent crystal in which atoms have $\mathrm{sp}^{3}$-type hybridization. The covalent bonds between atoms with $\mathrm{sp}^{3}$-type of hybridization can explain polytypism in $\mathrm{SiC}$. If a cubic crystal to orient along the crystallographic direction (111) and hexagonal and rhombohedra crystals along the (0001), the crystal of silicon carbide can be considered as a layered macromolecular one (like cyclohexane).

Cubic crystal $3 \mathrm{C}-\mathrm{SiC}$ is characterized by the interlayer structures like "N". Hexagonal $2 \mathrm{H}$ polytype layers are linked like "V" structures. The structure of another hexagonal and rhombohedra polytypes is determined by sequence of " $\mathrm{N}$ " and " $\mathrm{V}$ " structures along the $(0001)$ direction. The difference of a crystal structure of the silicon carbide polytypes is only in a nature of the interlayer bond, but structure of each layer is exactly the same.

From the fact that the energy of bonds between atoms in the cubic 3C-SiC (cubic arrangement) are not identical to the energy of bonds between atoms in hexagonal $2 \mathrm{H}-\mathrm{SiC}$ (hexagonal arrangement), it implies the assumption why $\mathrm{SF}_{i}$ spectrum has two parts $\mathrm{SF}-\mathrm{I}$ and SF - II. Namely, the difference in the interlayer bond's energies at the exactly same structure of each layer gives appearance of the super-fine structure of SF - I and SF - II parts in every spectrum.

These differences of the energy scale of SF - I and SF - II should be attributed to particular inter-atomic bonds. The phononless part of the SF-I spectrum consists of the components of radiative recombination, which is responsible for hexagonal and in the case of $\mathrm{SF}-\mathrm{II}$ for cubic arrangements of atoms.

The same character of all the $\mathrm{SF}_{i}$ spectra is confirmed by the same temperature behavior. Both of the $\mathrm{SF}_{i}$ spectra are observed at the temperatures 4.2 up to $35 \mathrm{~K}$ (Fig. 5). The parts $\mathrm{SF}-\mathrm{I}$ are observed at $4.2 \ldots 18 \mathrm{~K}$ (Fig. 5a), and $\mathrm{SF}-\mathrm{II}-$ at $4.2 \ldots 35 \mathrm{~K}$, accordingly (Fig. 5a, b). Fig. 5a illustrates the sequence decay of short-wave maximums for SF - I and SF - II (shown above in Fig. 3). At the temperatures higher than $18 \mathrm{~K}$, the sequence decay of short-wave maximums associated with $\mathrm{SF}-\mathrm{II}$ remains only (Fig. 5c). The thermal activation energy of the SF - I and SF - II parts is different and equal $E_{a}^{T} \approx 3.5 \mathrm{meV}$ and $E_{a}^{T} \approx 7.5 \mathrm{meV}$, accordingly (Fig. 5b).

The dependence of PL intensity with the intensity of exciting light has sub-linear character, namely: $I_{P L}=I_{\text {exc.light }}^{\alpha} \alpha=0.7$. At the same time, significant duration of the PL decay is observed. The PL intensity of the $\mathrm{SF}_{1}$ spectra is registered after switching-off the excitation and has the delay time $3 \cdot 10^{-3} \mathrm{~s}$ herewith $I_{P L}=0.02 I_{0}$. The intensity of the SF $-\mathrm{I}$ part decays rapidly. All these allow to make an assumption of possibility that there is a saturation effect of the radiation centers as well as the fact that recombined electrons and holes are significantly separated in space with the weak overlap of wave functions. High-temperature annealing $\left(T=2000{ }^{\circ} \mathrm{C}, t=5 \mathrm{~h}\right.$ ) of pure $\mathrm{SiC}$ crystals (with $\mathrm{SF}$ ) leads to a weakening the short-wave $\mathrm{SF}_{i}$ maximums.

In the beginning SF - I component disappears in all $\mathrm{SF}_{i}$ spectra (Fig. 3, curves 1 to 5), then the SF - II one disappears (Fig. 3, curves 7 to 10). After the hightemperature annealing for more than 5 hours, the SF - I components of spectra completely disappear. For better understanding the location of SF - I in the PL spectrum one, needs to study the overlap in PL spectra for $\mathrm{SiC}$ before (Fig. 6a, curve 1) and after deformation (Fig. 6a, curve 2).
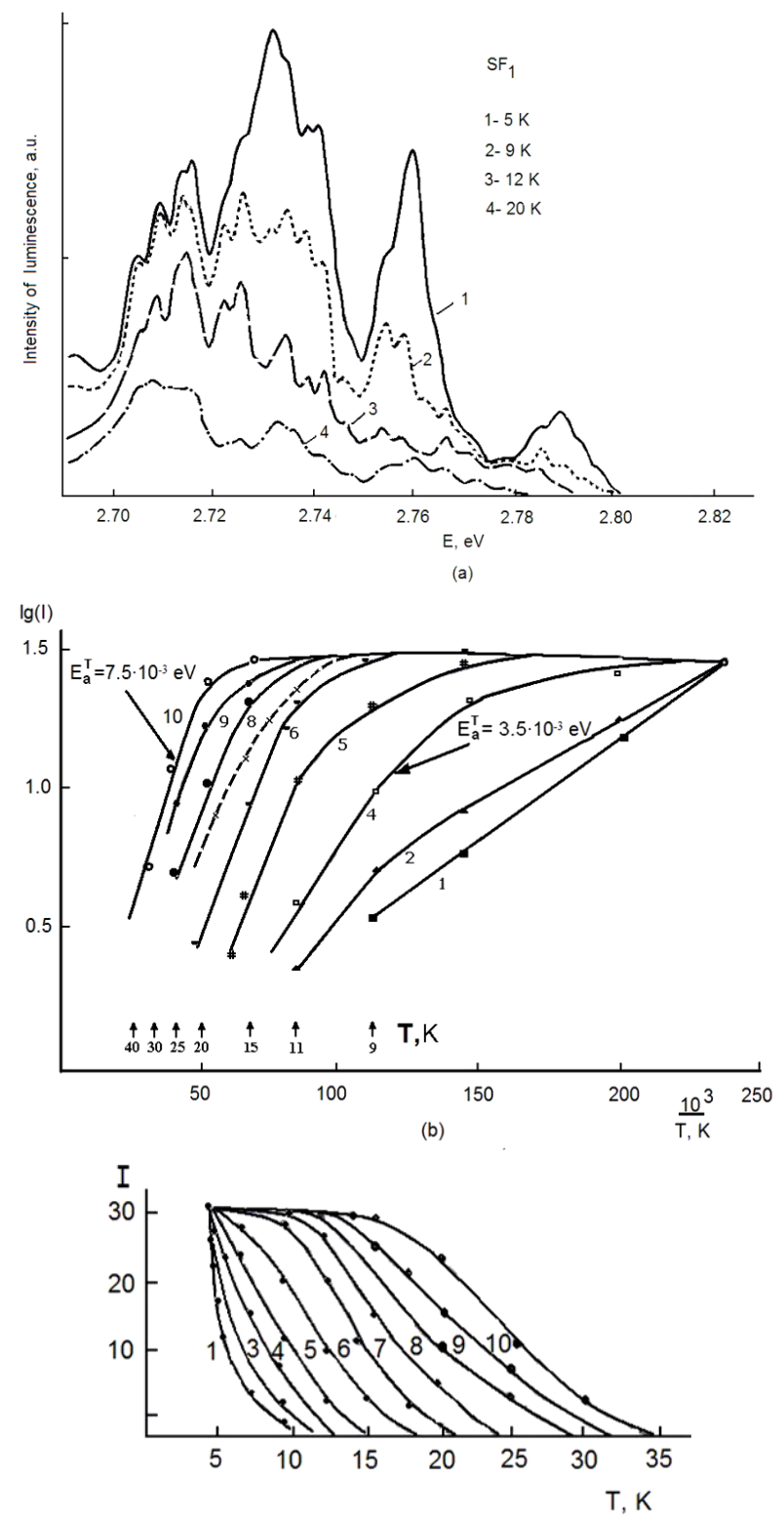

(c)

Fig. 5. Temperature behavior of the $\mathrm{SF}_{1}$ spectrum for the sample N5: (a) $\mathrm{SF}_{1}$ spectrum at various temperatures; (b) dependence of the PL intensity for the fine structure with inverse temperature; (c) temperature dependence of the PL intensity of the fine structure. 


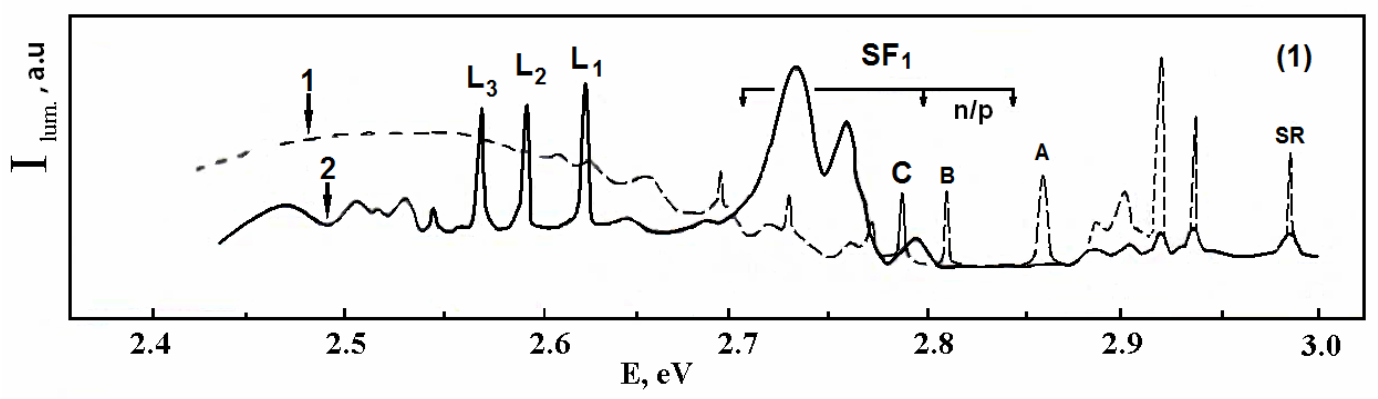

(a)

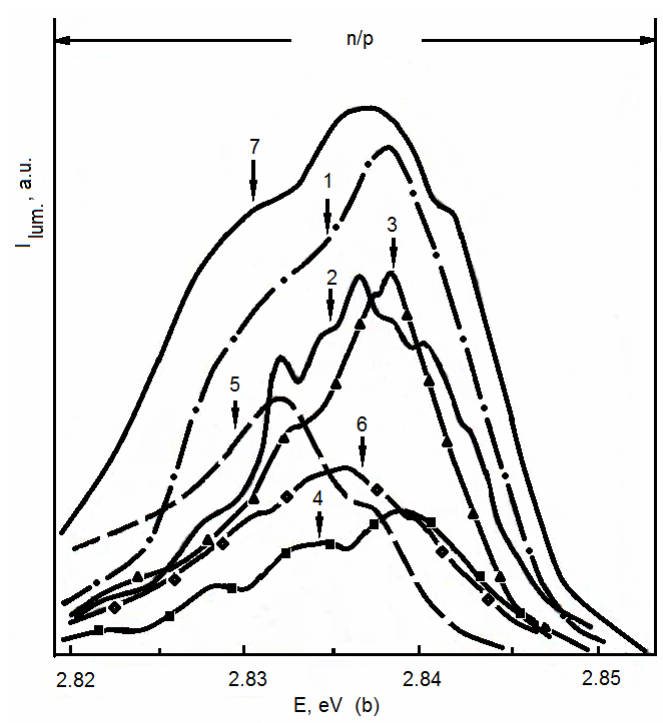

(b)

Fig. 6. LTPL spectra of SiC crystals: (a) pure $6 \mathrm{H} \mathrm{SiC}$ before and after plastic deformation; (b) intensity of the fine structure $\mathrm{SF}-\mathrm{I}$ component in $\mathrm{SF}_{\mathrm{i}}$ spectra of as-grown crystal polytype's joint and after plastic deformation by pressure.

The phononless part of $\mathrm{SF}_{1}$ spectra was obtained by calculation and is located between 2.793 and $2.852 \mathrm{eV}$ (Fig. 6a). Variation of changes in the distribution of intensities inherent to the phononless part of the fine $\mathrm{SiC}$ spectra is shown in Fig. 6b. The curves 1 and 2 in Fig. $6 \mathrm{~b}$ show the spectroscopic features of the two different samples of $\mathrm{SiC}$ growth polytype's joint. After plastic deformation of the non-defect $\mathrm{SiC}$ crystal (Fig. 6a, curve 1) by applying the force along c-axis, the $\mathrm{SF}-\mathrm{I}$ spectrum appears (Fig. 6b, curve 3). The appearance of the SF-I spectrum due to various external influences on the crystal is as follows: curve 4 (for the high force), curve 5 (for the low force), curve 6 (for additional applied forces). Curve 7 is the intensity distribution of $\beta \rightarrow \alpha$ transformation in the $\beta$-SiC after the high temperature annealing at $T=2000 \ldots 2100^{\circ} \mathrm{C}$. All these spectra emphasizes the complex structure of the SF - I spectrum and confirm the association with the peculiarities of structural states of the crystals. The superfine structure of SF - I component is related with the bond length between atoms in polytypes and depends on the crystallographic direction [21].

In order to understand the origin of super-fine structure in the spectrum, it needs to review the faulted- arrangement structure properties of different hexagonal layers in the $\mathrm{SiC}$ polytypes.

$\mathrm{SiC}$ polytypes have varying degrees of hexagonality, and the numeric value of which is defined as a ratio of amount of the atoms in the hexagonal arrangement and total amount of atoms in the polytype's cell. Similar phenomena were obtained in diamond and have a linear character of dependence of the interlayer distances with the degree of hexagonality [22]. The relationship of the degree of hexagonality and optical band gap in the $\mathrm{SiC}$ polytypes is linear [1]. By plotting the short-wave edge of each $\mathrm{SF}_{i}$ spectra on the energy scale and the corresponding excitation spectra in the dependence of the percentage hexagonality in the different polytypes, percentage of new hexagonality nanostructures can be obtained. The metastable form of nano-structures appear either in the growth process or after plastic deformation.

Short-wave edge location of the phononless part for each of the $\mathrm{SF}_{i}$ spectra is an indicator of the nanopolytype junction [23]. The locations of the spectrum the phononless parts of $\mathrm{SF}_{i}$ in the energy scale are summarized in Table.

\section{(C) 2013, V. Lashkaryov Institute of Semiconductor Physics, National Academy of Sciences of Ukraine}


Table. The phononless part of SF as indicator of formation of metastable intermediate phase.

\begin{tabular}{|l|c|c|c|}
\hline $\begin{array}{c}\text { Stacking } \\
\text { fault }\end{array}$ & $\begin{array}{c}\text { Locations } \\
(\mathrm{eV})\end{array}$ & Polytype & $\begin{array}{c}\text { Hexagonality } \\
(\%)\end{array}$ \\
\hline $\mathrm{SF}_{1}$ & 2.853 & $14 \mathrm{H}_{1}\langle 4334\rangle$ & 28.5 \\
\hline $\mathrm{SF}_{2}$ & 2.712 & $10 \mathrm{H}_{2}\langle 55\rangle$ & 20 \\
\hline $\mathrm{SF}_{3}$ & 2.611 & $14 \mathrm{H}_{2}\langle 77\rangle$ & 14.3 \\
\hline $\mathrm{SF}_{4}$ & 2.515 & $\mathrm{~N} / \mathrm{A}$ & 7 \\
\hline $\mathrm{SF}_{5}$ & 3.002 & $33 \mathrm{R}\left\langle(3332)_{3}\right\rangle$ & 37 \\
\hline $\mathrm{SF}_{6}$ & 2.78 & $8 \mathrm{H}\langle 44\rangle$ & 25 \\
\hline
\end{tabular}

After determining the position of the spectrum for the $\mathrm{SF}_{1}$ phononless part in the energy scale, it turned out that the short-wave part of the spectrum is the same to the position of the exciton band gap polytype $21 \mathrm{R}$ at $T=$ $4.2 \mathrm{~K}$, i.e. $2.853 \mathrm{eV}$.

The study of the excitation spectra gives better understanding the total complex panorama of the PL spectrum (SFs). Each SFs has its own spectrum of excitation. The total excitation spectrum repeats the absorption spectra. The linear dependence of the exciton band gap $\left(E_{g x}\right)$ with a percentage of hexagonality of polytypes indicates occurrence of a nanostructure.

If the initial polytypes were $15 \mathrm{R}\left\langle(23)_{3}\right\rangle$ and $6 \mathrm{H}$ $\langle 33\rangle$, then the spectrum of $\mathrm{SF}_{5}$ was observed. Due to the overlap with the other spectra, the $\mathrm{SF}_{4}$ spectrum is difficult to determine. $\mathrm{SF}_{4}$ corresponds to the unknown polytypes with lower percentage of hexagonality (up to $7 \%$ ). The $\mathrm{SF}_{6}$ had overlap with another spectrum and may correspond to the polytype $8 \mathrm{H}\langle 44\rangle$.

The possibility of occuring such new nano-phase with the percentage hexagonality less than $25 \%$ (both in the growth and in the result of solid state transformations) was confirmed by high-resolution electron microscopy [24, 25] and by first-principle study of $8 \mathrm{H}-, 10 \mathrm{H}-, 12 \mathrm{H}-, 18 \mathrm{H}-\mathrm{SiC}$ polytypes [26].

For example, the different percentage of hexagonality $(\mathrm{h})$ for structures $10 \mathrm{H}_{2}\langle 55\rangle$ and $10 \mathrm{H}_{1}$ $\langle 3223\rangle$ is shown in Fig. 7.

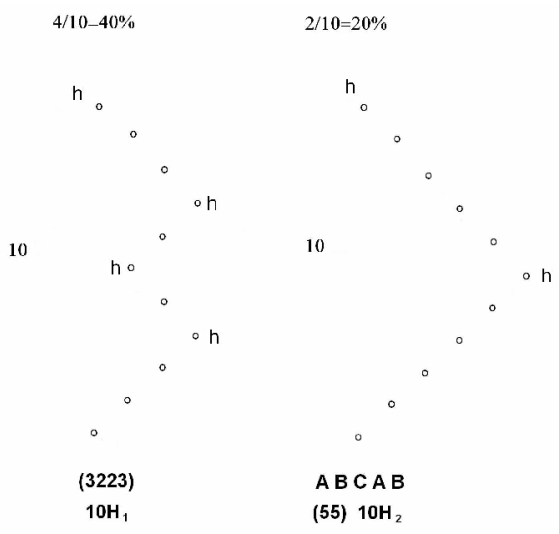

Fig. 7. Calculation of the hexagonality percentage for $10 \mathrm{H}-$ $\mathrm{SiC}$.
Moreover, the motif of the metastable nano-scale building structures $10 \mathrm{H}_{1}\langle 3223\rangle$ (with $40 \%$ hexagonality) corresponds to the motive of building structure $15 \mathrm{R}\left\langle(32)_{3}\right\rangle$, which takes place in stable conditions. The motif of $14 \mathrm{H}_{1}\langle 4334\rangle(28.5 \% \mathrm{~h})$ corresponds to the known stable polytype $21 \mathrm{R}\left\langle(34)_{3}\right\rangle$.

\section{Conclusion}

LTPL of pure $\alpha$-SiC crystals and pure crystals of $\beta$-SiC are represented by the similar spectra of SFs, which are indicators of formation of the metastable nanostructures, namely: $\quad 14 \mathrm{H} 1\langle 4334\rangle, \quad 10 \mathrm{H}_{2}\langle 55\rangle, \quad 14 \mathrm{H}_{2}\langle 77\rangle$, $33 \mathrm{R}\left\langle(3332)_{3}\right\rangle, 8 \mathrm{H}\langle 44\rangle$.

Comprehensive spectroscopic studies revealed the same principle of construction and the same behavior of each of the SFs spectra under various external influences on the crystals. The difference in interlayer bond's energies for the exactly same structure of each layer gives appearance of the super-fine SF-I and SF-II parts of every SFs spectrum. All SFs spectra are observed within the temperature range 4.2 to $35 \mathrm{~K}$ (SF $\mathrm{I}$ at $4.2 \ldots 15 \mathrm{~K}, \mathrm{SF}-\mathrm{II}$ at $4.2 \ldots 35 \mathrm{~K}) . \mathrm{SF}-\mathrm{I}$ corresponds to radiation caused by atoms creating the $\mathrm{V}$ (hexagonal) bonds between layers, SF - II corresponds to radiation of atoms creating the $\mathrm{N}$ (cubic) bonds between layers. Spectroscopic data have shown the same principle and the same behavior of the construction of each SFs spectrum under different external influences on the crystals.

The results of this work have shown the mechanism of interfacial rearrangements, which allows monitoring the processes of transforming the energy states in the crystal.

\section{References}

1. I.S. Gorban and G.N. Mishinova, The bases of luminescent diagnostic of dislocation structure of SiC crystals// Proc. SPIE, 3359, p. 187 (1998).

2. A. Galeckas, H.K. Nielsen, J. Linnros, A. Hallen, B.G. Svensson, and P. Pirouz, Investigation of stacking fault formation in hydrogen bombarded 4H-SiC // Mater. Sci. Forum, 483-485, p. 327-330 (2005).

3. S.I. Maximenko, P. Pirouz, and T. Sudarshan, Investigation of the electrical activity of partial dislocations in SiC p-i-n diodes // Appl. Phys. Lett. 87(3), 033503-0-033503-3 (2005).

4. A. Galeckas, and J. Linnros, P. Pirouz, Recombination induced stacking faults: Evidence for a general mechanism in hexagonal $\mathrm{SiC} / /$ Phys. Rev. Lett. 96(2), 025502-1-025502-4 (2006).

5. S.I. Maximenko, P. Pirouz and T.S. Sudarshan, Open core dislocations and surface energy of $\mathrm{SiC} / /$ Mater. Sci. Forum, 527-529, p. 439-442 (2006).

6. P. Pirouz, M. Zhang, H.McD. Hobgood, M. Lancin, J. Douin, and B. Pichaud, Nitrogen doping and 
multiplicity of stacking faults in SiC // Phil. Mag. $A, 86$ (29-31), p. 4685-4697 (2006).

7. G. Regula, M. Lancin, H. Idrissi, B. Pichaud and J. Douin, Structural characterization of double stacking faults induced by cantilever bending in nitrogen-doped 4H-SiC // J. Appl. Phys. 101(11), p. 113533.1-113533.5 (2007).

8. G.R. Fisher, P. Barnes, Toward a unified view of polytypism in silicon carbide // Phil. Mag. B, 61(2), p. 217-236 (1990).

9. S.I. Vlaskina, D.H. Shin, $6 \mathrm{H}$ to $3 \mathrm{C}$ polytype transformation in silicon carbide // Jpn. J. Appl. Phys. 38, p. L27-L29 (1999).

10. Shin Sugiyama, Motohiro Togaya, Phase relationship between $3 \mathrm{C}$ - and $6 \mathrm{H}$-silicon carbide at high pressure and high temperature // J. Amer. Ceram. Soc. 84 (12), p. 3013-3016 (2001).

11. S.I. Vlaskina // Semiconductor Physics, Quantum Electronics and Optoelectronics, 5(2), p. 252 (2002).

12. M. Durandurdu, $\mathrm{Ab}$ initio simulations of the structural phase transformation of $2 \mathrm{H}-\mathrm{SiC}$ at high pressure // Phys. Rev. B, 75, p. 235204 (2007).

13. S.W. Lee, S.I. Vlaskina, V.I. Vlaskin, I.V. Zaharchenko, V.A. Gubanov, G.N. Mishinova, G.S. Svechnikov, V.E. Rodionov, S.A. Podlasov, Silicon carbide defects and luminescence centers in current heated 6H-SiC // Semiconductor Physics, Quantum Electronics and Optoelectronics, 13(1), p. 24-29 (2010).

14. C. Raffy, J. Furthmuller, F. Bechstedt, Properties of interfaces between cubic and hexagonal polytypes of silicon carbide // J. Phys.: Condens. Matter, 14(48), p. 12725-12731 (2002).

15. A. Romano, J. Li, S. Yip, Atomistic simulation of rapid compression of fractured silicon carbide // $\mathrm{J}$. Nucl. Mater. 352, p. 22-28 (2006).

16. F. Shimojo, I. Ebbsjo, R.K. Kalia, A. Nakano, J.P. Rino, and P. Vashishta, Molecular-dynamics simulation of structural transformation in silicon carbide under pressure // Phys. Rev. Lett. 84, p. 3338-3341 (2000).

17. F. Bechstedt, P. Kackell // Phys. Rev. Lett. 75, p. 2180 (1995).

18. J.Q. Liu, M. Skowronski, C. Hallin, R. Soderholm and H. Lendenmann, Structure of recombinationinduced stacking faults in high voltage $\mathrm{SiC}$ p-n junctions // Appl. Phys. Lett. 80, p. 749 (2002).

19. M.S. Miao, S. Limpijumnong and W.R. Lambrecht, Stacking fault band structure in $4 \mathrm{H} \mathrm{SiC}$ and its impact on electronic devices // Appl. Phys. Lett. 79, p. 4360-4362 (2001).

20. S. Juillaguet, T. Robert, J. Camassel Optical investigation of stacking faults in $4 \mathrm{H}-\mathrm{SiC}$ epitaxial layers: Comparison of $3 \mathrm{C}$ and $8 \mathrm{H}$ polytypes // Mater. Sci. Eng. B - Solid State Mater. Adv. Technol., 165, p. 5-8 (2009).

21. A. Bauer, P. Reischauer, J. Kräusslich, N. Schell, W. Matz and K. Goetz, Structure refinement of the silicon carbide polytypes $4 \mathrm{H}$ and $6 \mathrm{H}$ : unambiguous determination of the refinement parameters // Acta Cryst. A, 57, p. 60-67 (2001).

22. B. Wen, J. Zhao, M. Bucknum, P. Yao, T. Li, First principles studies of diamond polytypes // Diamond Relat. Mater. 17, p. 356-364 (2008).

23. F. Herman, J.P. van Duke, R.L. Kortum, Electronic structure and spectrum of silicon carbide // Mater. Res. Bull. 4, p. S167-S178 (1969).

24. S. Shinozaki, K.R. Kisman, Aspects of "one dimensional disorder" in silicon carbide // J. Acta Metallurgica, 26, p. 769-776 (1978).

25. L.U. Ogbuji, T.E. Mitchell, A.H. Heuer, The $\beta \rightarrow \alpha$ transformation in polycrystalline $\mathrm{SiC} / / \mathrm{J}$. Amer. Ceram. Soc. 64(12), p. 91-99 (1981).

26. Kazuaki Kobayashi, Shojiro Komatsu, Firstprinciples study of $8 \mathrm{H}-, 10 \mathrm{H}-, 12 \mathrm{H}-$, and $18 \mathrm{H}-\mathrm{SiC}$ polytypes // J. Phys. Soc. Jpn. Appl. 024714 (2012). 